\title{
Comparative Analysis of Different Methods of Modeling the Thermal Effect of Circulating Blood Flow During RF Cardiac Ablation
}

\author{
A. González-Suárez* and E. Berjano
}

\begin{abstract}
Goal: Our aim was to compare the different methods of modeling the effect of circulating blood flow on the thermal lesion dimensions created by radiofrequency cardiac ablation and on the maximum blood temperature. Methods: Computational models were built to study the temperature distributions and lesion dimensions created by a non-irrigated electrode by two radiofrequency energy delivery protocols (constant voltage and constant temperature) under high and low blood flow conditions. Four methods of modeling the effect of circulating blood flow on lesion dimensions and temperature distribution were compared. Three of them considered convective coefficients at the electrode-blood and tissue-blood interfaces to model blood flow: 1) without including blood as a part of the domain; 2) constant electrical conductivity of blood; and 3) temperature-dependent electrical conductivity of blood $\left(+2 \% /{ }^{\circ} \mathrm{C}\right)$. Method 4$)$ included blood motion and was considered to be a reference method for comparison purposes. Results: Only Method 4 provided a realistic blood temperature distribution. The other three methods predicted lesion depth values similar to those of the reference method (differences smaller than $1 \mathrm{~mm}$ ), regardless of ablation mode and blood flow conditions. Conclusion: Considering the aspects of lesion size and maximum temperature reached in blood and tissue, Method 2 seems to be the most suitable alternative to Method 4 in order to reduce the computational complexity. Significance: Our findings could have an important implication in future studies of RF cardiac ablation, in particular, in choosing the most suitable method to model the thermal effect of circulating blood.
\end{abstract}

Index Terms-blood flow, cardiac ablation, catheter ablation computer modeling, radiofrequency ablation, thermal modeling.

\section{INTRODUCTION}

$\mathrm{C}$ OMPUTER modeling techniques have been used in numerous studies on radiofrequency (RF) catheter ablation (RFCA). The physical situation during this technique involves an active electrode (embedded at the tip of the

Manuscript received February 4th, 2015. This work was supported in part by the Spanish "Plan Estatal de Investigación, Desarrollo e Innovación Orientada a los Retos de la Sociedad" under Grant TEC2014-52383-C3 (TEC2014-52383-C3-1-R) and also by the Basque Government through the BERC 2014-2017 program and by Spanish Ministry of Economy and Competitiveness MINECO: BCAM Severo Ochoa excellence accreditation SEV-2013-0323.

E. Berjano and A. González-Suárez are with Biomedical Synergy, Electronic Engineering Department, Universitat Politècnica de València, València, Spain. *A. González-Suárez current affiliation is BCAM-Basque Center for Applied Mathematics, Bilbao, Basque Country, Spain (correspondence e-mail: agonzalezs@bcamath.org) percutaneous catheter) placed on the target zone of the cardiac tissue. RF current flows between this electrode and a large patch on the patient's back, but the thermal lesion is created exclusively in the target zone, due to the high current density being circumscribed to this zone. Since RFCA is always conducted inside the cardiac chamber, circulating blood is present in the scenario and is expected to have an effect on the created thermal lesion. The first RFCA theoretical model considered a spherical electrode completely surrounded by tissue, and hence did not take the circulating blood flow into account [1]. Two years later Labonté and Stuchly proposed the first RFCA computer model, which considered the thermal effect of circulating blood flow [2]. Their results suggested that heat loss due to circulating blood flow significantly influences the lesion geometry during RFCA [3,4]. From that time on all models have considered in one form or another the thermal effect of circulating blood. Some models do not consider blood as part of the model domain, and consequently the blood-tissue and blood-electrode interfaces were really model boundaries [3-8] on which an electrical boundary condition of zero current density was set. Other models consider blood as part of the domain [9-21], thus allowing RF current to flow through the blood. These models represent the initial value of the impedance (basal impedance) more realistically than those that do not include blood.

Both model types (with and without blood as part of the domain) include the thermal effect of the circulating blood flow by assuming a condition of forced thermal convection between the cardiac surface and circulating blood. The thermal convection approach requires two parameters to be set: blood temperature (always considered to be $37^{\circ} \mathrm{C}$ ) and a thermal convection coefficient, also known as convection film coefficient [22]. The tissue-blood and electrode-blood interfaces have different thermal convection coefficients $\left(h_{T}\right.$ and $h_{E}$ respectively) which have been estimated for different blood flow rates, depending on their location in the cardiac chamber. However, there is a wide variation in the values among the studies, and in fact Gallagher [23] has suggested some reasons for this, such as the pulsatile and turbulent nature of the blood flow, the changing value of the coefficient at the ablation site, and the high variability among patients and trials.

In models that include blood as part of the model, it is important to note that blood has a higher electrical conductivity $(\sigma)$ than cardiac tissue, and as a result RF current preferentially flows through blood, so that most of the heat is 
deposited there. This fact is even more marked when $\sigma$ is considered to increase with temperature, as it implies more heating of the blood, which is never realistic, since blood temperature remains more or less constant, around $37^{\circ} \mathrm{C}$. Using a thermal convection coefficient is not expected to offer an accurate temperature distribution in the blood. In 1999, Jain and Wolf [10] suggested that computer simulations in which the temperature-dependent electrical conductivity is only considered for the cardiac tissue but not for the blood could somehow compensate the positive feedback by which temperature in the blood increases unrealistically.

Subsequently, Jain and Wolf proposed the first RFCA model to take blood motion into account by estimating the fluid velocity field [24]. Their results suggested that including the fluid motion problem provides a realistic distribution of blood temperature and moreover is able to mimic the asymmetric temperature distribution in tissue and blood, where higher temperatures are observed on the outflow than the inflow side [24]. However, the inclusion of this additional problem means higher complexity and computational requirements. Surprisingly, in spite of not being able to use a convective boundary condition to model the blood temperature, they concluded that it provides a reasonable approximation of the temperature distribution in tissue in the case of constant voltage [24].

In conclusion, although some efforts have already been made to compare some of the different methods used to model the effect of the circulating blood flow, the comparisons only consider the case of constant voltage, and none has ever simultaneously included all the methods. The comparative performance under conditions of constant temperature remains unknown. Our objective was to compare simultaneously all the different methods proposed to date for modeling the effect of the circulating blood flow, specifically focusing on the blood and tissue temperature distributions obtained by each method for two RF power delivery modes (constant voltage and constant temperature) and two conditions of blood flow (high and low).

\section{METHODS}

\section{A. Description of the Model Geometry}

Fig. 1 shows the geometry and dimensions of the threedimensional computational model, which consists of a fragment of cardiac chamber filled with blood. Blood surrounds a non-irrigated active electrode, which is placed perpendicular to the cardiac surface. We considered an ablation electrode $7 \mathrm{Fr}$ in diameter and $4 \mathrm{~mm}$ long, as used in clinical practice. The electrode was assumed to be inserted into the tissue to a depth $\left(\mathrm{D}_{\mathrm{E}}\right)$ of $1.25 \mathrm{~mm}$ [14]. The model included a temperature sensor (thermistor $0.75 \times 0.3 \mathrm{~mm}$ ) embedded in the tip of the electrode, which was used for the temperature control simulation $[5,14,20]$. The dispersive electrode was modeled as an electrical boundary condition at a distance from the active electrode (bottom surface). Cardiac tissue thickness was $20 \mathrm{~mm}$ [24] and the dimensions of the fragment of cardiac chamber (X, Y and Z) were estimated by means of a convergence test in order to avoid boundary effects. In this test, the value of the maximal temperature achieved in the tissue $\left(\mathrm{T}_{\max }\right)$ after $60 \mathrm{~s}$ of RF heating was used as control parameter. We first considered a tentative spatial (i.e. minimum meshing size) and temporal resolution. To determine the appropriate parameters of $\mathrm{X}$ and $\mathrm{Y}(\mathrm{Z}=\mathrm{Y})$, we increased their values by equal amounts. When the difference in the $\mathrm{T}_{\max }$ between consecutive simulations was less than $0.5 \%$, we considered the former values to be adequate. We then determined adequate spatial and temporal resolution by means of similar convergence tests using the same control parameter as in the previous test. Discretization was spatially heterogeneous: the finest zone was always the electrode-tissue interface, where the largest voltage gradient was produced and hence the maximum value of current density. In the tissue, grid size was increased gradually with distance from the electrode-tissue interface.

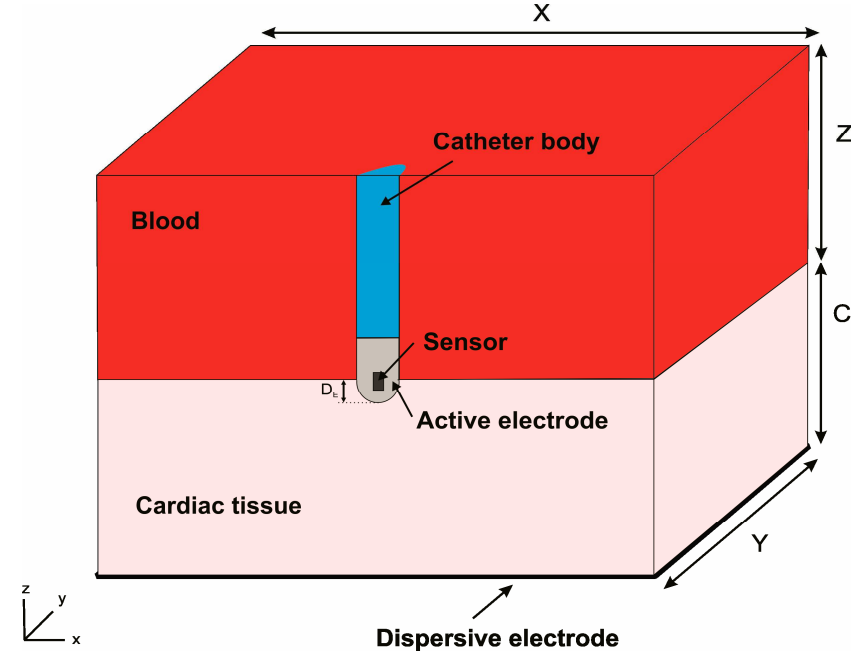

Fig. 1. Geometry of the computational models built for Methods 2, 3 and 4 (not to scale). Note that the XZ-plane is the symmetry plane in our model. Dimensions $\mathrm{X}$ and $\mathrm{Y}(\mathrm{Z}=\mathrm{Y})$ of the fragment of cardiac chamber filled with blood were obtained from a convergence test. The model geometry for Method 1 is identical, but without blood. RF current flows between a nonirrigated active electrode (7Fr, $4 \mathrm{~mm}$ ) and the dispersive electrode (bottom). The active electrode is assumed to be inserted into cardiac tissue to a depth $\mathrm{D}_{\mathrm{E}}$ $=1.25 \mathrm{~mm}$. The temperature sensor $(0.75 \times 0.3 \mathrm{~mm})$ is included in the model in order to implement the constant temperature protocol in which applied voltage is modulated to keep a constant temperature of $55^{\circ} \mathrm{C}$ in the sensor.

\section{B. Governing Equations}

The numerical models were based on a coupled electricthermal problem which was solved numerically using the Finite Element Method (FEM) and a PARDISO direct solver in COMSOL Multiphysics software (COMSOL, Burlington MA, USA). Simulations were run on a 64-bit PC with a 8processor Intel Xeon platform running at $2.67 \mathrm{GHz}$ with 48 GB of RAM. Time-dependent solutions were $60 \mathrm{~s}$ with a timestep of $0.05 \mathrm{~s}$.

The governing equation for the thermal problem was the Bioheat Equation modified by the enthalpy method [25,26], incorporating the phase change in order to model tissue vaporization: 
$\frac{\partial(\rho h)}{\partial t}=\nabla \cdot(k \nabla T)+q-Q_{p}+Q_{m}$

where $\rho$ was density $\left(\mathrm{kg} / \mathrm{m}^{3}\right), h$ enthalpy, $t$ time (s), $k$ thermal conductivity $(\mathrm{W} / \mathrm{m} \cdot \mathrm{K}), T$ temperature $\left({ }^{\circ} \mathrm{C}\right), q$ the heat source caused by RF power $\left(\mathrm{W} / \mathrm{m}^{3}\right), Q_{p}$ the heat loss caused by blood perfusion $\left(\mathrm{W} / \mathrm{m}^{3}\right)$ and $Q_{m}$ the metabolic heat generation $\left(\mathrm{W} / \mathrm{m}^{3}\right) . Q_{p}$ was not considered since it has been demonstrated both computationally [7] and experimentally [1] that the blood flow away the coronary artery does not have significant influence on the temperature distribution during RFCA. Likewise, $Q_{m}$ was not considered because it is negligible in comparison to the other terms during RFCA [3]. In biological tissues, enthalpy is related to temperature by the following expression [25,27]:

$\frac{\partial(\rho h)}{\partial t}=\frac{\partial T}{\partial t} \cdot \begin{cases}\rho_{l} c_{l} & 0<T \leq 99^{\circ} \mathrm{C} \\ H_{f_{g}} C & 99<T \leq 100^{\circ} \mathrm{C} \\ \rho_{g} c_{g} & T>100^{\circ} \mathrm{C}\end{cases}$

where $\rho_{i}$ and $c_{i}$ were the density and specific heat of cardiac tissue before phase-change (i.e. liquid tissue phase, $i=l$ ) and post-phase-change (i.e. gas tissue phase, $i=g$ ), respectively; $H_{f g}$ was the latent heat and $C$ the tissue water content. We considered a latent heat value of $2.162 \times 10^{9} \mathrm{~J} / \mathrm{m}^{3}$ which corresponds to the product of the water vaporization latent heat $(2257 \mathrm{~kJ} / \mathrm{kg})$ and the water density at $100^{\circ} \mathrm{C}\left(958 \mathrm{~kg} / \mathrm{m}^{3}\right)$, and the tissue water content inside the cardiac tissue (75\%).

At the RF frequencies $(\approx 500 \mathrm{kHz})$ used in RF heating and over the distance of interest, the biological medium can be considered almost totally resistive, since the displacement currents are much less important than conduction currents. A quasi-static approach was therefore possible to solve the electrical problem [28]. Then, the distributed heat source $q$ is given by $q=\sigma|\boldsymbol{E}|^{2}$, where $|\boldsymbol{E}|$ is the magnitude of the vector electric field $(\mathrm{V} / \mathrm{m})$ and $\sigma$ the electrical conductivity $(\mathrm{S} / \mathrm{m}) . \boldsymbol{E}$ is calculated from $\boldsymbol{E}=-\nabla \Phi$, where $\Phi$ is the voltage (V). The voltage is obtained by solving the Laplace equation, which is the governing equation of the electrical problem in absence of internal electric sources:

$\nabla \cdot(\sigma \nabla \Phi)=0$

\section{Properties of the Model Elements}

The thermal and electrical properties of the model elements (electrode, thermistor, plastic, blood and cardiac tissue) are shown in Table I $[13,14,29]$. The initial temperature in the entire model was $37^{\circ} \mathrm{C}$. The electrical $(\sigma)$ and thermal conductivity $(k)$ of cardiac tissue were temperature-dependent functions and were mathematically defined as piecewise functions (see Equation 4 and 5): For $\sigma$ we considered an exponential growth of $+1.5 \% /{ }^{\circ} \mathrm{C}$ up to $100^{\circ} \mathrm{C}$ [14], where $0.541 \mathrm{~S} / \mathrm{m}$ was the value of the electrical conductivity assessed at $37^{\circ} \mathrm{C}$ (see Table I), and then it decreased 4 orders for five degrees to model the tissue desiccation process [30]; and $k$ grew linearly $0.12 \% /{ }^{\circ} \mathrm{C}$ up to $100^{\circ} \mathrm{C}, 0.531 \mathrm{~W} / \mathrm{m} \cdot \mathrm{K}$ being the value of the thermal conductivity assessed at $37^{\circ} \mathrm{C}$ (see Table I), after which $k$ was kept constant [31].

$$
\begin{aligned}
\sigma(T) & =\left\{\begin{array}{lc}
0.541 e^{0.015(T-37)} & 0<T \leq 100^{\circ} C \\
1.371-0.274(T-100) & 100<T \leq 105^{\circ} C \\
1.371 \cdot 10^{-4} & T>105^{\circ} C
\end{array}\right. \\
k(T) & = \begin{cases}0.531+0.0012(T-37) & 0<T \leq 100^{\circ} C \\
0.606 & T>100^{\circ} C\end{cases}
\end{aligned}
$$

TABLE I

THERMAL AND ELECTRICAL CHARACTERISTICS OF THE ELEMENTS EMPLOYED

\begin{tabular}{|c|c|c|c|c|c|}
\hline \multicolumn{2}{|c|}{ Element/Material } & $\begin{array}{c}\sigma \\
(S / m) \\
\end{array}$ & $\begin{array}{c}\boldsymbol{k} \\
(\mathrm{W} / \mathbf{m} \cdot \mathbf{K}) \\
\end{array}$ & $\begin{array}{c}\rho \\
\left(\mathrm{kg} / \mathrm{m}^{3}\right) \\
\end{array}$ & $\begin{array}{c}c \\
\\
(\mathrm{~J} / \mathrm{kg} \cdot \mathbf{K}) \\
\end{array}$ \\
\hline \multicolumn{2}{|c|}{ Electrode/Pt-Ir } & $4.6 \times 10^{6}$ & 71 & 21500 & 132 \\
\hline \multicolumn{2}{|c|}{ Thermistor/Glass fiber } & $10^{-5}$ & 0.038 & 32 & 835 \\
\hline \multicolumn{2}{|c|}{ Catheter/Polyurethane } & $10^{-5}$ & 0.026 & 70 & 1045 \\
\hline \multicolumn{2}{|c|}{ Cardiac Chamber/Blood } & 0.667 & 0.541 & 1000 & 4180 \\
\hline \multirow{2}{*}{$\begin{array}{c}\text { Myocardium/ } \\
\text { Cardiac } \\
\text { tissue }\end{array}$} & $\begin{array}{l}\text { Liquid } \\
\text { phase }\end{array}$ & \multirow{2}{*}{0.541} & \multirow{2}{*}{0.531} & 1060 & 3111 \\
\hline & $\begin{array}{c}\text { Gas } \\
\text { phase }\end{array}$ & & & 370.44 & 2155.92 \\
\hline
\end{tabular}
IN THE NUMERICAL MODELS $[13,14,29]$

$\sigma$ : electric conductivity; $k$ : thermal conductivity; $\rho$ : density; $c$ : specific heat.

\section{Boundary Conditions}

Fig. 2a shows the electrical boundary conditions. A Dirichlet voltage boundary condition was applied at the active electrode surface. We considered two RF power delivery protocols: constant voltage and constant temperature. For constant voltage, the active electrode was fixed at $25 \mathrm{~V}$ for a period of $60 \mathrm{~s}$. For constant temperature, we implemented a proportional-integral (PI) control algorithm [32] using MATLAB (MathWorks, Natick, MA, USA) and the applied voltage was modulated to maintain the temperature in the sensor at $55^{\circ} \mathrm{C}$ [14]. All the outer surfaces of the model except the bottom surface were fixed to zero electric flux (Neumann boundary condition). The voltage on the bottom surface was set to $0 \mathrm{~V}$ (dispersive electrode) to mimic a monopolar configuration where RF current was forced to flow between the active and dispersive electrodes. We also conducted additional simulations to study the effect of the location of the dispersive electrode. We considered a new case in which the dispersive electrode was on the top surface of the model, as in [11]. In this way, the surface electrode was identical in size to the bottom surface of the model, so that only the effect of location was really being assessed.

Fig. $2 b$ and $2 c$ show the thermal and velocity boundary conditions. The effect of blood circulating inside the cardiac chamber was modeled by four methods which have been used in previous studies [3,5,9-11,13-15,17,20,24]. Each case was assessed under conditions of high and low blood flow. In all cases, a null thermal flux was used on the symmetry plane and a constant temperature of $37^{\circ} \mathrm{C}$ was fixed on the outer surfaces of the model at a distance from the ablating electrode (this was also the initial temperature value). 
(a)

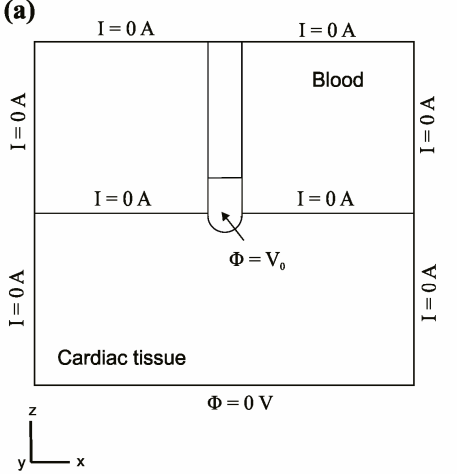

(b)

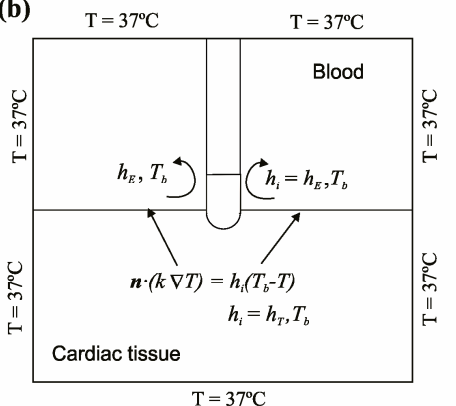

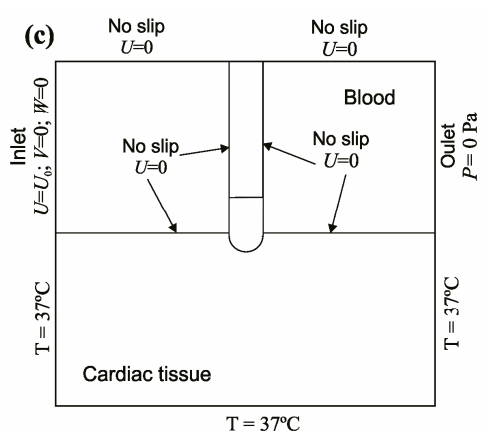

Fig. 2. Electrical (a) and thermal (b) and velocity (c) boundary conditions of the model: for Methods 1,2 and 3 and for Method 4 (c). $h_{E}$ and $h_{T}$ are the thermal convection coefficients at the electrode-blood and the tissue-blood interfaces, respectively. There is a specific value of these coefficients for high and low blood flow (see text for more details). Note that the model for Method 1 is without blood.

\section{E. Methods for Modeling the Effect of Circulating Blood Flow}

Method 1: The model geometry is similar to Fig. 1 but without including blood. The thermal effect of circulating blood flow was modeled using the thermal convective coefficients $h_{E}$ and $h_{T}$ (Figure $2 \mathrm{~b}$ ) as follows:

$\boldsymbol{n} \cdot(k \nabla T)=h_{i}\left(T_{b}-T\right)$

where $h_{i}$ is the thermal convective coefficient $\left(\mathrm{W} / \mathrm{m}^{2} \cdot \mathrm{K}\right)$ at the electrode-blood $(i=E)$ or tissue-blood interface $(i=T)$ and $T_{b}$ the blood temperature $\left(37^{\circ} \mathrm{C}\right)$. The thermal convective coefficient at the tissue-blood interface $\left(h_{T}\right)$ was calculated as [14]:

$u=\left(\frac{h_{T}}{h_{r e f}}\right)^{1.25} u_{r e f}$

where $u$ is blood velocity in cardiac chamber, $u_{\text {ref }}(24 \mathrm{~cm} / \mathrm{s})$ a reference blood velocity measured at specific location inside the cardiac chamber [20] and $h_{r e f}\left(=1417 \mathrm{~W} / \mathrm{m}^{2} \cdot \mathrm{K}\right)$ the reference convective coefficient obtained from $u_{\text {ref. }}$ We obtained two values of $h_{T}$ for low and high blood flow: 265 and $610 \mathrm{~W} / \mathrm{m}^{2} \cdot \mathrm{K}$ for $u$ of 3 and $8.5 \mathrm{~cm} / \mathrm{s}$ respectively [24]. The thermal convective coefficient at the electrode-blood interface $\left(h_{E}\right)$ was calculated as:

$h_{E}=\frac{N u k_{b}}{d}$

where $N u$ is the Nusselt number, $k_{b}$ the blood thermal conductivity $(\mathrm{W} / \mathrm{m} \cdot \mathrm{K})$ and $\mathrm{d}$ the catheter diameter $(7 \mathrm{Fr}=2.33$ $\mathrm{mm})$. The Nusselt number can be approximated as follows:

$N u=0.683 \operatorname{Re}^{0.466} \operatorname{Pr}^{0.333}$

where Re and Pr are the Reynolds and the Prandtl numbers, which are calculated using (10) and (11):
$\operatorname{Re}=\frac{\rho_{b} u d}{\mu}$
$\operatorname{Pr}=\frac{c_{b} \mu}{k_{b}}$

where $c_{b}$ is the specific heat of blood $(\mathrm{J} / \mathrm{kg} \cdot \mathrm{K})$ and $\mu$ the viscosity of the blood $\left(2.1 \times 10^{-3} \mathrm{~kg} /(\mathrm{m} \cdot \mathrm{s})\right)$ [14]. We obtained two $h_{E}$ for low and high flow of blood velocity: 2059 and 3346 $\mathrm{W} / \mathrm{m}^{2} \cdot \mathrm{K}$ for $u$ of 3 and $8.5 \mathrm{~cm} / \mathrm{s}$ respectively [24].

Method 2: The model geometry corresponds exactly with Fig. 1, i.e. the blood is included in the domain. In this method, electrical conductivity of blood was assumed to be constant, as in previous studies [3,9,11,13-15]. As in Method 1, convective boundary conditions were applied at the electrode-blood and tissue-blood interfaces to simulate cooling by the blood flow (Fig. 2b). The values of $h_{E}$ and $h_{T}$ were identical to Method 1 .

Method 3 is identical to Method 2 except for the fact that the electrical conductivity of the blood was considered to be temperature-dependent with a change rate of $+2 \% /{ }^{\circ} \mathrm{C}[10,17]$.

Method 4: The model geometry is also identical to that shown in Fig. 1. Here we directly consider the blood motion in the model [24] instead of using convective boundary conditions. We considered a laminar fluid flow, plus the high and low flow velocities mentioned above [24]. In this case, there is a bidirectional thermal-flow coupled problem, i.e. the incompressible Navier-Stokes equation from fluid dynamics works together with the heat transfer equation. Therefore, the so-called advection term [33] was included on the right of (1), which represents the heat loss due to blood flow:

$$
\frac{\partial(\rho h)}{\partial t}=\nabla \cdot(k \nabla T)+q-Q_{p}+Q_{m}-\rho c u \cdot \nabla T
$$

where $\boldsymbol{u}(\mathrm{x}, \mathrm{y}, \mathrm{z})$ is the blood velocity field $(\mathrm{m} / \mathrm{s})$ and is derived from the incompressible Navier-Stokes equation, which consists of a Momentum equation (momentum principle or balance of forces) and a Mass equation (mass conservation):

$$
\begin{aligned}
& \rho \frac{\partial \boldsymbol{u}}{\partial t}+\rho \boldsymbol{u} \cdot \nabla \boldsymbol{u}=-\nabla P+\mu \nabla^{2} \boldsymbol{u}+\boldsymbol{F} \\
& \nabla \cdot \boldsymbol{u}=0
\end{aligned}
$$


where $P$ is the pressure (Pa), $\mu$ the viscosity of the blood (the same value as mentioned in Method 1) and $\boldsymbol{F}\left(F_{x}, F_{y}, F_{z}\right)$ the volume of forces $\left(\mathrm{N} / \mathrm{m}^{3}\right)$, which was absent in this model.

Fig. 2c shows the velocity boundary conditions applied in Method 4. A no slip condition (nonpermeable surface, i.e. the fluid at the wall was not moving) was applied on the upper surfaces of the fluid volume, at the symmetry plane (plane XZ in Fig. 1) and at the tissue-blood and electrode-blood interfaces. An inlet boundary condition of velocity type was applied to the left surface of the fluid volume to simulate the two flow velocities (in $x$-direction), while an outlet boundary condition of zero pressure/no viscous stress was fixed on the right surface of the fluid volume.

\section{F. Assessment of the Thermal Lesion Dimensions and Comparison Criterion}

As in previous studies, we used the $50^{\circ} \mathrm{C}$ isotherm as the thermal lesion contour $[10,11,13,14,17,20]$. Although Wood et al. concluded that the lethal isotherm for cardiac myocardium is near $61^{\circ} \mathrm{C}$ [34], their staining procedure to measure the area of acute necrosis has been criticized for underestimating the extent of irreversible tissue injury [35]. Consequently, the range between $50-56^{\circ} \mathrm{C}$ is usually considered to faithfully represent the isotherm of irreversible myocardial injury in hyperthermic ablation.

We compared the thermal lesion dimensions obtained, in particular the maximum width (W) and depth (D) created in the cardiac tissue. The maximum temperature values reached in the tissue and blood ( $\mathrm{T}_{\text {max_tissue }}$ and $\mathrm{T}_{\text {max_blood }}$ respectively) in all four methods were also compared. Since Method 4 provides the most realistic modeling approach, this was considered as the reference method, and the results from the other three methods were compared to it. We considered that the differences in lesion depth and width were insignificant for values lower than $1 \mathrm{~mm}$, since this value is approximately that of the deviation $( \pm 0.5 \mathrm{~mm})$ observed in experimental RFCA studies with a non-irrigated electrode [22,36]. Likewise, the differences in $\mathrm{T}_{\text {max } \_ \text {tissue }}$ or $\mathrm{T}_{\text {max blood }}$ were considered to be insignificant for values lower than $4^{\circ} \mathrm{C}$, since this value is approximately that of the observed deviation $\left( \pm 2^{\circ} \mathrm{C}\right)[22]$.

\section{RESULTS}

\section{A. Building the Models}

After the convergence tests conducted with the computational model, we obtained the following optimum values: dimensions $\mathrm{X}=80 \mathrm{~mm}$ and $\mathrm{Y}=40 \mathrm{~mm}(\mathrm{Z}=\mathrm{Y})$, grid size of $0.2 \mathrm{~mm}$ in the finest zone (electrode-tissue interface), and step time of $0.05 \mathrm{~s}$.

\section{B. Spatial Distributions of Temperature in Tissue and Blood}

Fig. 3 shows the temperature distribution in cardiac tissue and blood after $60 \mathrm{~s}$ of RFA considering constant voltage (Fig. 3a) and temperature control (Fig. 3b) for the four methods considered. The solid black line indicates the $50^{\circ} \mathrm{C}$ isothermal borderline and therefore outlines the thermal lesion contour. Table II and III shows the lesion dimensions, the maximum temperature reached in cardiac tissue and blood, and applied energy for the cases shown in Fig. 3.

With both ablation protocols, temperature distribution in the blood was symmetrical around the electrode axis for Methods 2 and 3 (where blood flow motion was not taken into account), while with Method 4 (reference method) the blood temperature was higher on the outflow side of the electrode. In the same way, the temperature distribution in the cardiac tissue was only asymmetrical for Method 4, especially with the constant voltage protocol. This can be noted as the isothermal line was extended to the outflow side of the electrode.

\section{Constant Voltage Ablation Mode}

Blood temperatures $\left(\mathrm{T}_{\text {max_blood }}\right)$ computed with Method 4 were considerably lower than those from Methods 2 and 3 (note that Method 1 does not include blood and hence $\mathrm{T}_{\text {max_blood }}$ cannot be computed). $\mathrm{T}_{\text {max_blood }}$ values from Method 4 were $\approx 90^{\circ} \mathrm{C}$ for low and $\approx 80^{\circ} \mathrm{C}$ for high blood flow. In contrast, Method 2, in which blood $\sigma$ was assumed to be constant, provided values of Tmax_blood lower than Method 3 (blood $\sigma$ is temperature-dependent): $108.7^{\circ} \mathrm{C}$ vs. $137.8^{\circ} \mathrm{C}$ for low and $95.4^{\circ} \mathrm{C}$ vs. $125.9^{\circ} \mathrm{C}$ for high blood flow. Tissue temperature $\left(\mathrm{T}_{\text {max }}\right.$ tissue $)$ was similar between the four methods under high blood flow conditions (values ranging from 100 to $105^{\circ} \mathrm{C}$ ). In contrast, in the case of low blood flow, Methods 1 , 2 and 3 provided $\mathrm{T}_{\text {max } \_ \text {tissue }}$ values $5-7^{\circ} \mathrm{C}$ lower than Method 4 $\left(103-106^{\circ} \mathrm{C}\right.$ vs. $\left.111^{\circ} \mathrm{C}\right)$.

All Methods provided lesions of similar depth (differences lower than $1 \mathrm{~mm}$ ), and only Method 3 provided higher values for lesion width as compare to Method 4: $2.5 \mathrm{~mm}$ and $1.8 \mathrm{~mm}$ bigger with low and high blood flow, respectively. The total amount of energy (E) applied during ablation can explain the performance of each Method. Regardless of blood flow, Methods 2 and 4 applied a similar amount of energy (525-543 J), while Method 1 applied much less energy (251-265 J), and Method 3 rather more (765-825 J).

\section{Constant Temperature Ablation Mode}

Blood temperatures $\left(\mathrm{T}_{\text {max_blood }}\right)$ computed with Method 4 were also lower than with Methods 2 and 3. $\mathrm{T}_{\text {max_blood }}$ values from Method 4 were $\approx 60^{\circ} \mathrm{C}$ for low and $63.5^{\circ} \mathrm{C}$ for high blood flow. Differences between $\mathrm{T}_{\text {max_blood }}$ values from Methods 2 and 3 compared to Method 4 were significantly lower with constant temperature mode than with constant voltage mode. In contrast, differences between $T_{\text {max }}$ tissue values from Methods 2 and 3 compared to Method 4 under high blood flow conditions were significantly higher with constant temperature mode than with constant voltage. $\mathrm{T}_{\text {max } \_ \text {tissue }}$ values from Methods 2 and 3 provided similar results to Method 4 for low blood flow. Method 1 provided $\mathrm{T}_{\text {max } \_ \text {tissue }}$ values significantly higher than Method 4, regardless of blood flow, and this fact was possibly related to having values of lesion width 1-1.7 mm larger than Method 4. Methods 2 and 3 had similar lesion width values to Method 4. All methods provided similar depth lesions (differences lower than $1 \mathrm{~mm}$ ). Regardless of blood flow, Method 4 applied the highest amount of energy than the other methods. 
TABLE II

LESION DIMENSIONS (DEPTH D AND MAXIMUM WIDTH W), MAXIMUM TEMPERATURE REACHED IN THE TISSUE ( $\mathrm{T}_{\text {MAX_TISSUE }}$ ) AND BLOOD (T ENERGY (E) AFTER $60 \mathrm{~S}$ OF RFA USING CONSTANT VOLTAGE $(25 \mathrm{~V})$

\begin{tabular}{c|c|c|c|c|c|c|c|c|c|}
\hline \hline & \multicolumn{4}{|c|}{ Low blood flow } & \multicolumn{4}{c}{ High blood flow } \\
\hline Methods $^{*}$ & 1 & 2 & 3 & 4 & 1 & 2 & 3 & 4 \\
\hline $\mathrm{D}(\mathrm{mm})$ & 7.71 & 7.70 & 8.36 & 7.64 & 7.47 & 7.69 & 8.06 & 7.53 \\
\hline $\mathrm{W}(\mathrm{mm})$ & 12.84 & 13.31 & $\mathbf{1 5 . 3 3}$ & 12.81 & 11.72 & 12.49 & $\mathbf{1 3 . 7 2}$ & 11.92 \\
\hline $\mathrm{T}_{\text {max_tissue }}\left({ }^{\circ} \mathrm{C}\right)$ & $\mathbf{1 0 5 . 9}$ & $\mathbf{1 0 6 . 0}$ & $\mathbf{1 0 2 . 9}$ & 111.3 & 104.4 & 105.0 & 100.0 & 102.4 \\
\hline $\mathrm{T}_{\text {max_blood }}\left({ }^{\circ} \mathrm{C}\right)$ & - & $\mathbf{1 0 8 . 7}$ & $\mathbf{1 3 7 . 8}$ & 89.6 & - & $\mathbf{9 5 . 4}$ & $\mathbf{1 2 5 . 9}$ & 79.9 \\
\hline $\mathrm{E}(\mathrm{kJ})$ & 0.265 & 0.543 & 0.825 & 0.536 & 0.251 & 0.539 & 0.765 & 0.525 \\
\hline \hline
\end{tabular}

TABLE III

LESION DIMENSIONS (DEPTH D AND MAXIMUM WIDTH W), MAXIMUM TEMPERATURE REACHED IN THE TISSUE ( $\mathrm{T}_{\text {MAX_tISSUE }}$ ) AND BLOOD (T $\mathrm{T}_{\text {MAX_BLOOD }}$ ), AND APPLIED ENERGY (E) AFTER $60 \mathrm{~S}$ OF RFA CONSIDERING CONSTANT TEMPERATURE $\left(55^{\circ} \mathrm{C}\right)$

\begin{tabular}{c|c|c|c|c|c|c|c|c}
\hline \hline & \multicolumn{3}{|c|}{ Low blood flow } & \multicolumn{4}{c}{ High blood flow } \\
\hline Methods $^{*}$ & 1 & 2 & 3 & 4 & 1 & 2 & 3 \\
\hline $\mathrm{D}(\mathrm{mm})$ & 6.24 & 4.85 & 4.70 & 5.32 & 7.21 & 5.72 & 5.63 \\
\hline $\mathrm{W}(\mathrm{mm})$ & $\mathbf{1 0 . 0 5}$ & 8.17 & 8.29 & 8.05 & $\mathbf{1 1 . 2 9}$ & 9.06 & 9.08 \\
\hline $\mathrm{T}_{\text {max_tissue }}\left({ }^{\circ} \mathrm{C}\right)$ & $\mathbf{8 8 . 5}$ & $\mathbf{6 8 . 8}$ & $\mathbf{6 5 . 8}$ & 73.7 & $\mathbf{1 0 2 . 5}$ & $\mathbf{7 8 . 9}$ & $\mathbf{7 3 . 8}$ \\
\hline $\mathrm{T}_{\text {max__blood }}\left({ }^{\circ} \mathrm{C}\right)$ & - & 63.9 & $\mathbf{6 6 . 7}$ & 59.5 & - & $\mathbf{6 9 . 6}$ & $\mathbf{7 5 . 7}$ \\
\hline $\mathrm{E}(\mathrm{kJ})$ & 0.139 & 0.193 & 0.220 & 0.248 & 0.210 & 0.273 & 0.32 \\
\hline \hline
\end{tabular}

Method 4 is considered to be reference method for comparison purposes. In bold those values of temperature and thermal lesion sizes disagreeing more than $4^{\circ} \mathrm{C}$ and $1 \mathrm{~mm}$ respectively with respect to Method 4 (reference method).

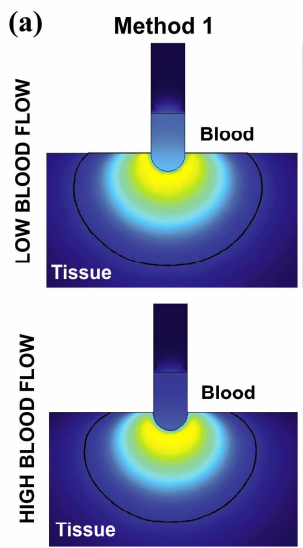

(b)
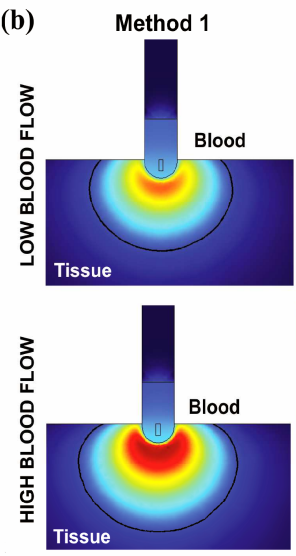

Method 2
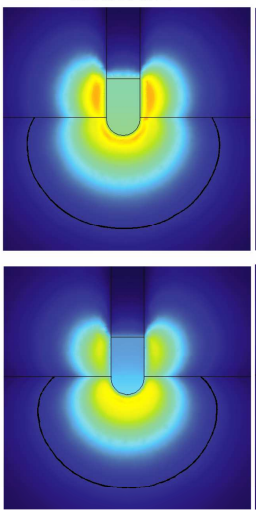

Method 2
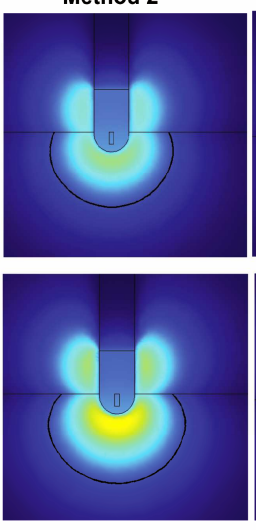

Method 3
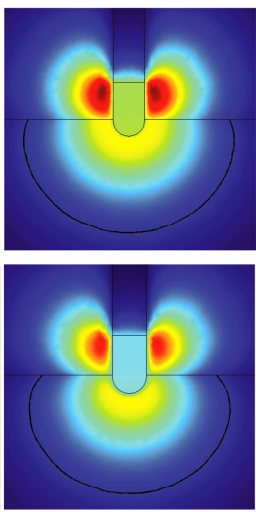

Method 3
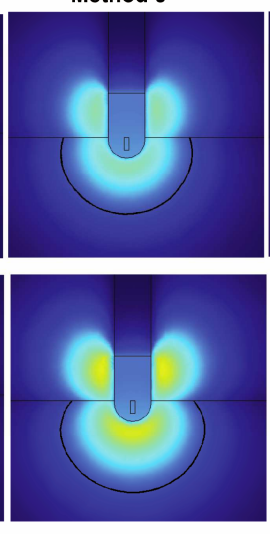

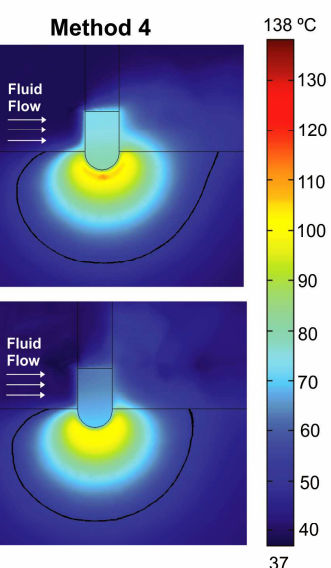

Method 4
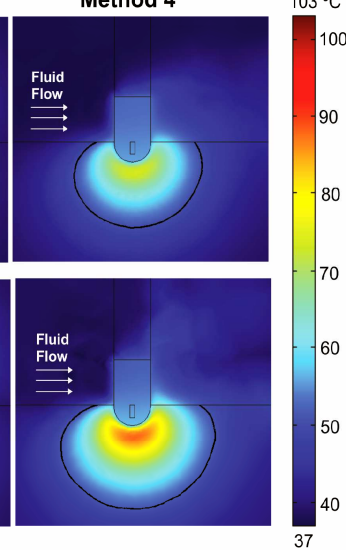

Fig. 3. Temperature distributions in the cardiac tissue and blood after $60 \mathrm{~s}$ of RFA, considering constant voltage (a) and constant temperature (b) for the four methods of modeling the effect of circulating blood flow, and for low and high flow conditions. The solid black line corresponds to the $50^{\circ} \mathrm{C}$ isotherm. 


\section{E. Overall Assessment}

In this study Method 4 was assumed to be a reference method since theoretically it provided more realistic values of blood and tissue temperatures. The differences between the values computed from Method 4 and the others can therefore be considered as a measurement of the error of each method.

In an effort to assess all the five considered outcomes together (lesion depth and width, blood and tissue temperatures, and amount of applied energy), we calculated the absolute value of the difference between the outcomes computed from Method 4 and each of the other methods, and plotted the results in a radar chart as shown in Fig. 4 to provide a sort of performance metrics of Methods 1, 2 and 3. This allowed us to evaluate the suitability of each method graphically and easily, since the relative position of each point from the center is related to its error. A shorter distance to the center thus means a smaller difference with respect to the results computed with Method 4. The inclusion of Method 1 in the general analysis is questionable, since it is not able to provide blood temperature values. In Fig. $4 a-b$ it can be seen that for the constant voltage ablation mode, Method 3 values are furthest from Method 4, regardless of blood flow. Method 2 in general shows the best fit with Method 4. In the case of constant temperature ablation mode, Fig. 4c-d shows that Method 1, which performs well in the voltage-constant ablation mode, now offers the worst performance, along with being unable to compute blood temperature. It is important to note that Method 2 performs better than Method 3, especially for low blood flow.

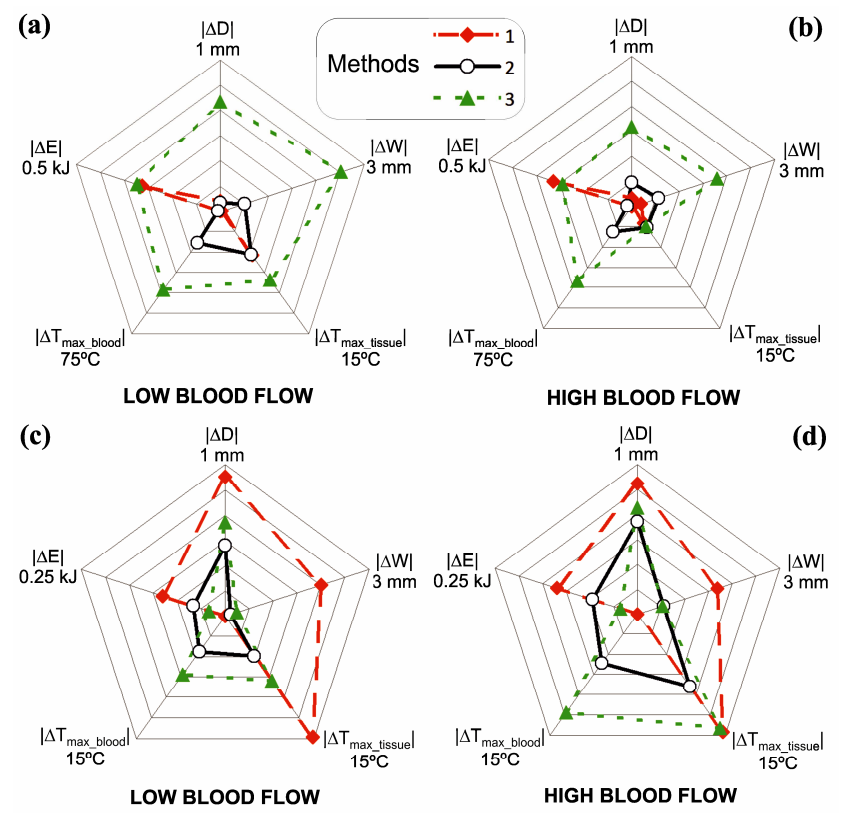

Fig. 4. Radar charts with absolute differences between outcomes computed from Method 4 and those computed from Methods 1, 2 and 3 for constant voltage ( $a$ and $b$ ) and constant temperature ( $c$ and d) ablation mode, and for low (a and $\mathrm{c}$ ) and high (b and d) blood flows. Lesion depth $(|\Delta \mathrm{D}|)$, lesion width $(|\Delta \mathrm{W}|)$, blood $\left(\left|\Delta \mathrm{T}_{\text {max_blood }}\right|\right)$ and tissue $\left(\mid \Delta \mathrm{T}_{\text {max_tissue }}\right)$ temperatures, and amount of applied energy $(|\Delta \mathrm{E}|)$

\section{F. Effect of Short Ablations}

The temperature distribution in cardiac tissue and blood, and the lesion shapes created in the tissue after $30 \mathrm{~s}$ of RFA (results not shown) largely coincided with those shown in Figure 3. However, we did note some differences within the first 10 seconds of heating (see Figure 5): 1) regarding the lesion shape with constant voltage, Method 4 showed a practically symmetrical temperature distribution around the electrode axis; 2) the lesion sizes were almost identical for all methods and ablation protocols.

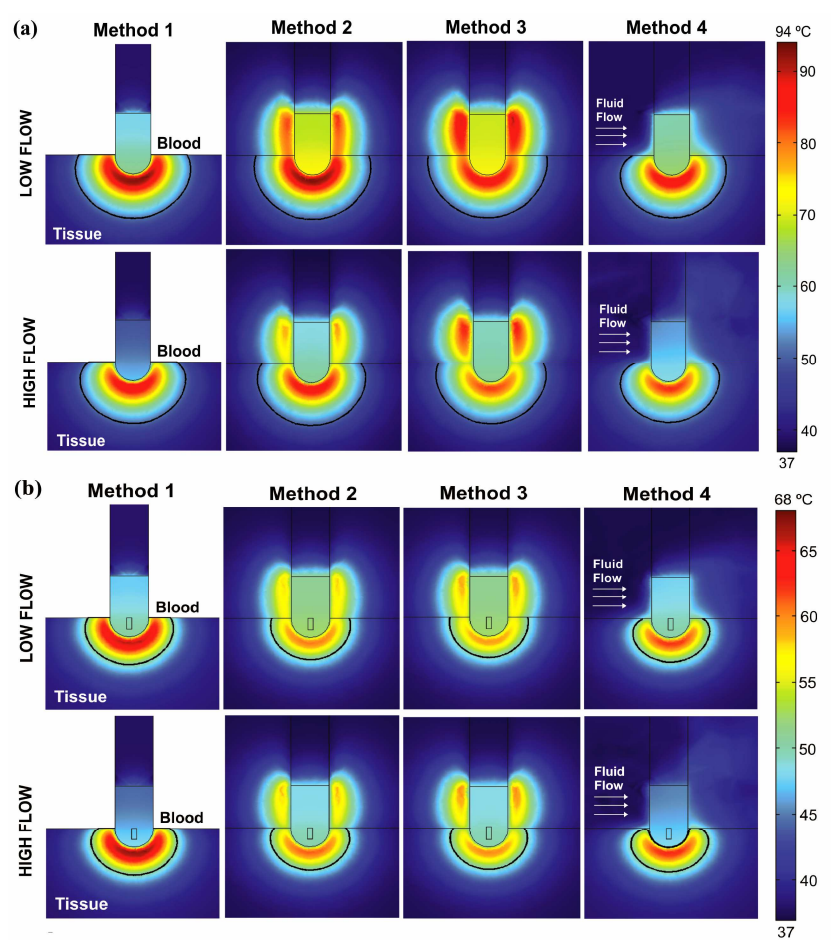

Fig. 5. Temperature distributions in the cardiac tissue and blood in the first seconds of RFA (at $10 \mathrm{~s}$ ), considering constant voltage (a) and constant temperature (b) for the four methods of modeling the effect of circulating blood flow, and for low and high flow conditions. The solid black line corresponds to the $50^{\circ} \mathrm{C}$ isotherm.

\section{G. Effect of Dispersive Electrode Location}

When the dispersive electrode was located on the top surface of the model, only Methods 2, 3 and 4 could be simulated, since the Method 1 model did not include this surface (corresponding to the blood surface). We observed that the lesion shapes were similar with the dispersive electrode in the top and bottom positions for both ablation protocols. The lesion dimensions (depth and width) were smaller in the top than in the bottom position, regardless of ablation protocol. However, the reduced size of the lesion dimensions with respect to the bottom position (optimal) was more pronounced with constant voltage than with constant temperature, especially using Method 3 and high blood flow: $1.3 \mathrm{~mm}$ in depth and $1.7 \mathrm{~mm}$ in width for constant voltage, whereas the size was reduced by $0.4 \mathrm{~mm}$ in depth and $0.3 \mathrm{~mm}$ in width for constant temperature. The temperature in the tissue was also reduced with the dispersive electrode in the top position for the two ablation protocols. This drop in temperature was also 
more pronounced with constant voltage than with constant temperature, especially with Method 4, considering low blood flow: 8.8 and $0.4^{\circ} \mathrm{C}$ for constant voltage and constant temperature, respectively.

On the other hand, the effect of changing the dispersive electrode position on blood temperature and energy differed with the ablation protocol used. Both blood temperature and energy rose with the dispersive electrode in the top position at constant temperature, whereas these parameters were seen to fall with the constant voltage protocol. The differences with respect to the bottom position were always greater at constant voltage than constant temperature, especially when using Method 3. Blood temperature was reduced by $12.7^{\circ} \mathrm{C}$ using constant voltage for Method 3 and low blood flow, while it rose by $1^{\circ} \mathrm{C}$ for the same case at constant temperature. With respect to the energy for the same case (Method 3 and low blood flow), the drop was $103 \mathrm{~J}$ using constant voltage, while it rose by $13 \mathrm{~J}$ at constant temperature.

\section{DISCUSSION}

\section{A. Comparative Analysis of the Methods}

Method 4, considered as the reference method for comparison purposes, was able to provide a realistic temperature distribution in the blood, with higher values on the outflow side of the electrode (see Fig. 3). The other methods tested provided symmetrical distributions. These findings had already been reported for constant voltage ablation mode [24], but not for constant temperature ablation mode. Our results confirm the same performance in the case of constant temperature mode.

The blood temperatures values obtained with Method 4 were lower than those from the other three methods, and the differences were higher in the case of constant voltage. Our findings also suggest that the differences between methods are considerable in ablations longer than 10 seconds, or in other words, the mathematical mechanisms responsible for providing different solutions between the four methods increase in importance with longer times.

Our computer results are in agreement with those obtained by [11], since they confirm that locating the dispersive electrode in front of the ablating electrode (i.e. on the bottom surface of the model) produces larger lesions (both in depth and width) and higher temperatures in the cardiac tissue than locating it behind the tip (i.e. in the top surface of the model), considering both the constant voltage and constant temperature protocols.

Since Methods 1, 2 and 3 do not include the blood motion problem, they are easier than Method 4 in terms of computational complexity. It is therefore crucial to determine which of them could be used instead of Method 4 to achieve similar computational results in terms of lesion size, and blood and tissue temperatures. The conditions of this equivalence have to be stated in each case, i.e. according to the ablation mode used and blood flow conditions. For instance, our results show that all Methods (1, 2 and 3) predict similar values of lesion depth (differences smaller than $1 \mathrm{~mm}$ ), regardless of ablation mode and blood flow conditions. Method 3, in which electrical conductivity of blood is temperature-dependent, offers a worse prediction of lesion depth for constant voltage, as suggested by Jain and Wolf [10]. However, this prediction is somewhat better in the case of constant temperature (see Figs. 4c and 4d). Furthermore, our results show that Method 1, the simplest one because blood is not included in the model domain, offers a better prediction of lesion depth than Method 3 in the case of constant voltage, but not in the case of constant temperature. Similarly, Method 3 fails to predict the lesion width in the case of constant voltage (differences higher than $1 \mathrm{~mm}$ with Method 4), while Method 1 fails in the case of constant temperature (see Fig. 4). In short, in order to predict lesion depth and width, Methods 1 and 2 seems to be suitable in the case of constant voltage, while Methods 2 and 3 would be suitable for constant temperature ablation.

Surprisingly, despite the potential of Methods 1, 2 and 3 to predict reasonably well both lesion depth and width, all offer a poor estimate of the maximum temperatures reached in the tissue $\left(\mathrm{T}_{\text {max }}\right.$ tissue $)$. Only for voltage-constant ablation mode and high blood flow, all methods offer values of $\mathrm{T}_{\text {max }}$ tissue similar to Method 4. If all circumstances are taken into account (two ablation modes and two blood flow conditions), Method 2 performs best. Finally, as regards to predicting the maximum temperatures reached in the blood ( $\left.\mathrm{T}_{\text {max_blood }}\right)$, only Methods 2 and 3 are reasonable, and once more Method 2 shows lower prediction errors. Note that the axis scale of $\mathrm{T}_{\max \text { blood }}$ is different in cases of constant voltage (Fig. 4a and b) and constant temperature (Fig. $4 \mathrm{c}$ and d) and prediction errors are much smaller in the case of constant temperature.

It is interesting to observe the results of Method 1 in detail. For the constant temperature mode, Method 1 has a poor performance (see dashed lines in Fig. 4c and d), but for constant-voltage ablation, it offers low prediction errors for lesion size and $\mathrm{T}_{\text {max_tissue }}$ (similar to Method 2). The only difference with Method 1 is the amount of applied energy, around half of that used in Method 4. This is precisely the cause of the good performance of Method 1 under constantvoltage conditions: since the blood is not included in the model domain, the total impedance with Method 1 is significantly higher than with the other methods (blood, whose electrical conductivity is even higher than cardiac tissue, is absent). The delivered current, and hence applied power, is lower. In other words, although in Method 1 all the RF power is exclusively applied to the cardiac tissue, which could imply better heating and hence bigger lesions, the high impedance value allows two opposing factors to be balanced. Moreover, since Method 1 excludes blood from the model domain, this method would be really modeling a blood-free scenario, as actually happens in surgical RF cardiac ablation (epicardial ablation on a pumping heart or endocardial ablation on a stopped heart).

If one takes all the items into account (lesion size and maximum temperature reached in blood and tissue), Method 2 seems to be the most suitable alternative, since it provides similar values to Method 4. Interestingly, the four methods offered lesion sizes almost identical in the first seconds (see 
Fig. 5), which suggests that the differences are larger over time.

Finally, the computational time required to solve the models with Method 2 was about 7 times less than that required by Method 4. For example, the computer simulation of the model using temperature constant ablation protocol and high blood flow took almost 3 hours with Method 4 and around 25 minutes with Method 2. This finding is surely relevant for future theoretical RFCA models when blood is not included in the model domain, and also in order to assess retrospectively the results from previous computational studies.

\section{B. Comparison with Experimental Results}

Only the study by Jain and Wolf [24] provides solid results on the appropriateness of Method 4 as compared to Method 2 (the best alternative in terms of predicting thermal lesion size) for the case of constant voltage. By comparing their computer and experimental results, Jain and Wolf demonstrated that both Methods were able to predict the experimental temperatures in the tissue with a mean error of less than $6^{\circ} \mathrm{C}$. This is in line with our results using constant voltage, since Methods 2 and 4 provided lesion depths and widths similar to within $1 \mathrm{~mm}$ (see Table II). On the other hand, in order to predict the temperature distribution in the fluid, Jain and Wolf [24] reported that while Method 4 showed a mean error $<7^{\circ} \mathrm{C}$ (compared to their experimental results), Method 2 had a mean error of as much as $40^{\circ} \mathrm{C}$. All this suggests that Method 4 would be the only method able to predict blood temperature during a constant voltage ablation.

In the case of constant temperature, Matsudaira et al [37] found experimentally that temperature distribution in the blood was clearly non symmetrical around the electrode axis, with higher values on the side opposite the blood flow. They also found that the maximal temperature in the blood was $68.3 \pm 4.3^{\circ} \mathrm{C}$ with a $4-\mathrm{mm}$ electrode after 60 seconds of ablation, $55^{\circ} \mathrm{C}$ target temperature, and high blood flow condition. Although this temperature value is reasonably close to the values of $\mathrm{T}_{\text {max_blood }}$ obtained by us with Methods 2 and 4 $\left(69.6^{\circ} \mathrm{C}\right.$ and $63.5^{\circ} \mathrm{C}$, respectively), only Method 4 was able to reproduce the asymmetry in blood temperature around the electrode axis, as observed experimentally by Matsudaira et al [37].

Regarding the thermal lesion dimensions using constant temperature, our computer results are comparable to those obtained by Balázs et al [39] using an ex vivo experimental model. Although their aim was to compare the performance of gold- and platinum-iridium-tip RF electrodes, they used 4-mm non irrigated electrodes with a constant temperature mode $\left(50^{\circ} \mathrm{C}\right.$ target) for $60 \mathrm{~s}$, which was very similar to the conditions of our computer simulations. They obtained thermal lesions $5.75 \mathrm{~mm}$ in depth and $10.00 \mathrm{~mm}$ in width, which are approximately in agreement with those obtained by us for constant temperature $\left(55^{\circ} \mathrm{C}\right.$ target) by Methods 2,3 and 4 (see Table III). In fact, our results suggest that any of these three methods can suitably predict lesion dimensions in the case of constant temperature. The comparison with results by Balázs et al [39] suggests that our model, regardless of the method used, predicts lesion depth and width reasonably well. The difference in applied energy between these authors' [39] experimental results $(0.92 \mathrm{~kJ})$ and our simulations $(0.35 \mathrm{~kJ})$ was possibly due to the higher flow used in their experiments $(50 \mathrm{~cm} / \mathrm{s}$ vs. $8.5 \mathrm{~cm} / \mathrm{s})$. It is well known that the presence of blood flow increases the RF power required to maintain the same target temperature [37]. Additionally, we can compare our computer results in the case of constant temperature with those obtained by Jain et al [11] using an in vivo model. They found thermal lesions $5.8 \pm 0.8 \mathrm{~mm}$ in depth and $9.3 \pm 1.9 \mathrm{~mm}$ in width, which are also very close to our results, even though Jain et al [11] employed a slightly higher target temperature $\left(60^{\circ} \mathrm{C}\right.$ instead of $\left.55^{\circ} \mathrm{C}\right)$.

It is almost impossible to compare the values of $\mathrm{T}_{\text {max }}$ tissue obtained from our simulations to those from experimental studies, since the maximum tissue temperature is reached $\approx 1$ $\mathrm{mm}$ under the electrode (see Fig. 3), while tissue temperatures are usually measured at depths of 3 and $7 \mathrm{~mm}$ [37]. However, Method 4 was the only method able to provide a non symmetrical temperature distribution around the ablating electrode axis - note that the surface width of the thermal lesion computed in the tissue was considerably greater on the blood outflow side (see Fig. 3). This is also in agreement with the experimental results obtained by Jain and Wolf [38] and Matsudaira et al [37], who found that the temperature reached in the cardiac tissue on the outflow side was greater than on the inflow side.

In conclusion, the comparison between our computer results and the experimental results from previous studies suggests that: 1) the proposed model with Methods 2 and 4 allows the thermal lesion dimensions to be realistically reproduced, and 2) Method 4 in particular allows the temperature distributions in the blood to be reproduced.

\section{Limitations of the Study}

The constant power mode was not considered in this study. This mode is usually employed with irrigated electrodes and not with dry electrodes, as modeled here. In spite of this, it was observed that for a $15-\mathrm{W} 60$-s ablation with dry electrodes the applied voltage does not vary more than $2 \mathrm{~V}$ throughout ablation [10], and consequently constant voltage and constant power can be considered to be comparable in the context of this study.

\section{CONCLUSIONS}

Only Method 4 seems to provide a realistic blood temperature distribution. By assuming this method as a reference method, our findings suggest that the three methods considered are able to predict values of lesion depth similar to those of the reference method (differences smaller than 1 $\mathrm{mm}$ ), regardless of ablation mode and blood flow conditions. Finally, bearing all the items in mind (lesion size and maximum temperature reached in blood and tissue), Method 2 seems to be the most suitable alternative to Method 4 in terms of reducing the computational complexity. 


\section{REFERENCES}

[1] D. E. Haines and D. D. Watson, "Tissue heating during radiofrequency catheter ablation: a thermodynamic model and observations in isolated perfused and superfused canine right ventricular free wall," Pacing Clin. Electrophysiol., vol. 12, pp. 962-976, Jun. 1989.

[2] S. Labonté and S. S. Stuchly, "Radio-frequency ablation of the myocardium: a numerical model," in Annual International Conference of the IEEE Engineering tn Medicine and Biology Society, 1991, vol. 13 , no. 2, pp. 619-620.

[3] S. Labonté, "Numerical model for radio-frequency ablation of the endocardium and its experimental validation," IEEE Trans. Biomed. Eng., vol. 41, no. 2, pp. 108-115, Feb. 1994.

[4] S. Labonté, "A Computer Simulation of Radio-Frequency Ablation of the Endocardium," IEEE Trans. Biomed. Eng., vol. 41, no. 9, pp. 883 890, Sep. 1994

[5] J. Alba-Martínez et al., "Could it be advantageous to tune the temperature controller during radiofrequency ablation? A feasibility study using theoretical models," Int. J. Hyperth., vol. 27, no. 6, pp. 539548, 2011.

[6] F. Hornero and E. Berjano, "Esophageal Temperature During Radiofrequency Catheter Ablation of Left Atrium: A Three-Dimensional Computer Modeling Study," J. Cardiovasc. Electrophysiol., vol. 17, no. 4, pp. 405-410, Apr. 2006

[7] M. Obradović et al., "Finite element modeling of the endocardial radiofrequency ablation," J. Serbian Soc. Comput. Mech., vol. 4, no. 2 , pp. 43-53, 2010.

[8] R. T. Roper and M. R. Jones, "Benchmark Solution for the Prediction of Temperature Distributions During Radiofrequency Ablation of Cardiac Tissue," J. Biomech. Eng., vol. 126, no. 4, pp. 519-522, Aug. 2004.

[9] A. González-Suárez et al., "Radiofrequency cardiac ablation with catheters placed on opposing sides of the ventricular wall: Computer modelling comparing bipolar and unipolar modes," Int. J. Hyperth., vol. 30, no. 6, pp. 372-384, Sep. 2014.

[10] M. K. Jain and P. D. Wolf, "Temperature-Controlled and ConstantPower What Affects Lesion Growth?," IEEE Trans. Biomed. Eng., vol. 46, no. 12, pp. 1405-1412, Dec. 1999.

[11] M. Jain et al., "Effect of Skin Electrode Location on Radiofrequency Ablation Lesions: An In Vivo and a Three-Dimensional Finite Element Study," J. Cardiovasc. Electrophysiol., vol. 9, no. 12, pp. 1325-1335, Dec. 1998.

[12] Y. Lai et al., "Lesion size estimator of cardiac radiofrequency ablation at different common locations with different tip temperatures," IEEE Trans. Biomed. Eng., vol. 51, no. 10, pp. 1859-1864, Oct. 2004.

[13] D. Panescu et al., "Three-dimensional Finite-Element Analysis of Current-Density and Temperature Distributions During Radiofrequency Ablation," IEEE Trans. Biomed. Eng., vol. 42, no. 9, pp. 879-890, Sep. 1995

[14] D. Schutt et al., "Effect of electrode thermal conductivity in cardiac radiofrequency catheter ablation : A computational modeling study," Int. J. Hyperth., vol. 25, no. 2, pp. 99-107, Mar. 2009.

[15] A. V. Shahidi and P. Savard, "A finite element model for radiofrequency ablation of the myocardium," IEEE Trans. Biomed. Eng., vol. 41, no. 10, pp. $963-968$, Oct. 1994

[16] E. J. Woo et al., "A New Catheter Design Using Needle Electrode for Subendocardial RF Ablation of Ventricular Muscles: Finite Element Analysis and in vitro Experiments," IEEE Trans. Biomed. Eng., vol. 47, no. 1, pp. 23- 31, Jan. 2000.

[17] S. Tungjitkusolmun et al., "Thermal-electrical finite element modelling for radio frequency cardiac ablation: effects of changes in myocardial properties," Med. Biol. Eng. Comput., vol. 38, no. 5, pp. 562-568, Sep. 2000.

[18] S. Tungjitkusolmun et al., "Modeling Bipolar Phase-Shifted Multielectrode Catheter Ablation," IEEE Trans. Biomed. Eng., vol. 49, no. 1, pp. 10-17, Jan. 2002.

[19] S. Tungjitkusolmun et al., "Finite Element Analyses of Uniform Current Density Electrodes for Radio-Frequency Cardiac Ablation," IEEE Trans. Biomed. Eng., vol. 47, no. 1, pp. 32-40, Jan. 2000.

[20] S. Tungjitkusolmun et al., "Guidelines for Predicting Lesion Size at Common Endocardial Locations During Radio-Frequency," IEEE Trans. Biomed. Eng., vol. 48, no. 2, pp. 194-201, Feb. 2001.

[21] N. Gallagher et al., "Contact Geometry Affects Lesion Formation in Radio-Frequency Cardiac Catheter Ablation," PLoS One, vol. 8, no. 9 , pp. 1-10, Sep. 2013
[22] H. Cao et al., "Flow effect on lesion formation in RF cardiac catheter ablation," IEEE Trans. Biomed. Eng., vol. 48, no. 4, pp. 425-433, Apr. 2001.

[23] N. P. Gallagher, "Radio-frequency catheter ablation for treatment of atrial fibrillation: The influence of probe contact on impedance and lesion formation," M.S. thesis, Dept. Electrical and Computer Eng., Calgary Univ., Calgary, Alberta, Canada, 2012.

[24] M. K. Jain and P. D. Wolf, "A three-dimensional finite element model of radiofrequency ablation with blood flow and its experimental validation," Ann. Biomed. Eng., vol. 28, no. 9, pp. 1075-1084, Sep. 2000.

[25] J. P. Abraham and E. M. Sparrow, "A thermal-ablation bioheat model including liquid-to-vapor phase change, pressure- and necrosisdependent perfusion, and moisture-dependent properties," Int. J. Heat Mass. Tran., vol. 50, no. 13-14, pp. 2537-2544, 2007.

[26] J. Byeongman and A. Alptekin, "Prediction of the extent of thermal damage in the cornea during conductive keratoplasty," J. Therm. Biol., vol. 35, no. 4, pp. 167-174, May. 2010.

[27] G. Zhao et al., "Effect of blood flow and metabolism on multidimensional heat transfer during cryosurgery," Med. Eng. Phys., vol. 29, no. 2, pp. 205-215, Mar. 2007.

[28] J. D. Doss, "Calculation of electric fields in conductive media," Med. Phys., vol. 9, no. 4, pp. 566-573, Jul.-Aug. 1982.

[29] T. Pätz et al., "Simulation of radiofrequency ablation including water evaporation," in IFMBE Proceedings of the World Congress on Medical Physics and Biomedical Engineering 25/IV , pp. 1287-1290, 2009.

[30] D. Haemmerich et al., "Hepatic radiofrequency ablation with internally cooled probes: effect of coolant temperature on lesion size," IEEE Trans. Biomed. Eng., vol. 50, no. 4, pp. 493-499, Apr. 2003.

[31] E. J. Berjano, "Theoretical modeling for radiofrequency ablation: stateof-the-art and challenges for the future," Biomed. Eng. Online, 5:24, Apr. 2006.

[32] D. Haemmerich and J. G Webster, "Automatic control of finite element models for temperature-controlled radiofrequency ablation," Biomed. Eng. Online, 4:42, Jul. 2005.

[33] F. P. Incropera et al., "Fundaments of Heat and Mass Transfer," 6th Ed. Hoboken: John Wiley \& Sons, 2006.

[34] M. Wood et al., "Direct measurement of the lethal isotherm for radiofrequency ablation of myocardial tissue," Circ. Arrhythm. Electrophysiol., vol. 4, no. 3, pp. 373-37, Jun. 2011.

[35] D. E. Haines, "Letter by Haines regarding article: Direct measurement of the lethal isotherm for radiofrequency ablation of myocardial tissue," Circ. Arrhythm. Electrophysiol., vol. 4, no. 5, pp. e67, Oct. 2011.

[36] M. A. Wood et al., "Effect of electrode orientation on lesion sizes produced by irrigated radiofrequency ablation catheters," J. Cardiovasc. Electrophysiol., vol. 20, no. 11, pp. 1262-1268, Nov. 2009.

[37] K. Matsudaira et al., "High incidence of thrombus formation without impedance rise during radiofrequency ablation using electrode temperature control," Pacing Clin. Electrophysiol., vol. 26, no. 5, pp. 1227-1237, May. 2003.

[38] M. K. Jain and P. D. Wolf, "In vitro temperature map of cardiac ablation demonstrates the effect of flow on lesion development," Ann. Biomed. Eng., vol. 28, no. 9, pp. 1066-1074, Sep. 2000.

[39] T. Balázs et al., "Ablation time efficiency and lesion volume - in vitro comparison of $4 \mathrm{~mm}$, non irrigated, gold- and platinum-iridium-tip radiofrequency ablation catheters," J. Interv. Card. Electrophysiol., vol. 36, no. 1, pp. 13-18, Jan. 2013. 